\title{
MicroRNA-216a inhibits the growth and metastasis of oral squamous cell carcinoma by targeting eukaryotic translation initiation factor $4 \mathrm{~B}$
}

\author{
LEI LI and HUI-QIANG MA \\ Department of Stomatology, Jining No. 1 People's Hospital, Jining, Shandong 272011, P.R. China
}

Received August 17, 2014; Accepted March 23, 2015

DOI: $10.3892 / \mathrm{mmr} .2015 .3761$

\begin{abstract}
There is increasing evidence to suggest that microRNAs (miRNAs; miRs) are involved in the development of oral squamous cell carcinoma (OSCC). miR-216a has been identified as being involved in tumorigenesis, however, the mechanisms of miR-216a in various types of cancer, either as a tumor suppressor or as an oncogenic miRNA, and the specific regulatory role of $\mathrm{miR}-216 \mathrm{a}$ in OSCC remain to be elucidated. The present study demonstrated that the expression of miR-216a was significantly reduced in OSCC tissues and cell lines. Overexpression of miR-216a significantly suppressed the proliferation, colony formation, migration and invasion of the OSCC cells. In addition, eukaryotic translation initiation factor 4B (EIF4B) was identified as a direct target of miR-216a, which was observed to be upregulated in the OSCC tissues. Furthermore, overexpression of EIF4B significantly attenuated the antitumor effect of miR-216a, and a negative correlation was observed between miR-216a and EIF4B in the OSCC tissues. Taken together, these findings indicated that miR-216a has a suppressive role in OSCC cells by directly targeting EIF4B, and may function as a potential prognostic biomarker and novel therapeutic target.
\end{abstract}

\section{Introduction}

Oral cancer is one of most common types of malignancy worldwide, and $\sim 42,440$ new cases were diagnosed in the United States in 2014 (1). Oral squamous cell carcinoma (OSCC) accounts for $\sim 90 \%$ of oral cancer cases. Despite significant advances in therapeutic strategies in the last few years, the overall 5-year survival rates of patients with OSCC is $\sim 60 \%$ at the age of 62 years (2). Funk et al (3) reported that the 5-year survival rate is almost $80 \%$ in the early

Correspondence to: Dr Hui-Qiang Ma, Department of Stomatology, Jining No. 1 People's Hospital, 6 Jiankang Road, Jining, Shandong 272011, P.R. China

E-mail: mhq0313@sina.com

Key words: oral squamous cell carcinoma, microRNA-216a, eukaryotic translation initiation factor $4 \mathrm{~B}$, growth, metastasis stages, however, this rate decreases to between 20 and $40 \%$ in advanced-stage OSCC. This indicates that early detection is essential for improving the survival rates and prognosis in OSCC. Therefore, an improved understanding of the molecular biology and pathogenesis of OSCC is essential for the development of novel biomarkers and therapies.

MicroRNAs (miRNAs) are a class of small non-coding RNAs of $\sim 21-23$ nucleotides in length, which regulate target genes through the 3'-untranslated regions (3'-UTRs) to induce mRNA degradation and inhibit mRNA translation (4). Accumulating evidence has reported that miRNAs are important in tumorigenesis due to their aberrant expression (5), and can function either as oncogenes or tumor suppressors in various types of cancer, depending on their downstream target genes $(5,6)$. In addition, several miRNAs have been identified to be dysregulated, regulating the initiation and progression of OSCC (7). miR-216a has been observed to be downregulated and function as a tumor suppressor in several types of cancer (8-10). However, the role of miR-216a in the regulation of key genes and signaling pathways associated with OSCC remains to be elucidated.

In the present study, the expression levels of miR-216a in OSCC specimens and cell lines, and the effects on the growth and metastasis of OSCC cells were investigated. In addition, the present study aimed to identify whether eukaryotic translation initiation factor 4B (EIF4B) is a potential target of miR-216a and identify correlations with miR-216a in the OSCC tissues.

The current study may not only provide a novel understanding of the regulatory mechanism of miR-216a but additionally offer a novel target for the treatment of OSCC.

\section{Materials and methods}

OSCC specimens, cell lines and transfection. A total of 23 paired OSCC tissue samples and adjacent non-tumor tissue samples were collected from patients undergoing resection of OSCC at the No.1 People's Hospital (Jining, China) between June 2011 and December 2013. Of the patients, 15 were men and 8 were women, with a median age of 51 years (range, 22-83). No patients received chemotherapy or radiotherapy prior to surgery. The tissue samples were immediately snap frozen in liquid nitrogen and stored at $-80^{\circ} \mathrm{C}$. Histopathology was confirmed by two independent pathologists. The study was 
approved by the Hospital Ethical Committee and informed consent was obtained from each patient prior to commencement of the investigation.

The SCC-4 and CAL 27 cell lines were obtained from American Type Culture Collection (Manassas, VA, USA) and cultured in RPMI-1640 medium (GE Healthcare, Logan UT, USA). The HEK293 cells were obtained from the Type Culture Collection of the Chinese Academy of Sciences (Shanghai, China) and were maintained in Dulbecco's modified Eagle's medium. All the media were supplemented with $10 \%$ fetal bovine serum (FBS) at $37^{\circ} \mathrm{C}$ under $5 \% \mathrm{CO}_{2}$. For transfection, cells were transfected using Lipofectamine 2000 (Invitrogen Life Technologies, Carlsbad, CA, USA) according to the manufacturer's instructions. Following $4 \mathrm{~h}$ incubation at $37^{\circ} \mathrm{C}$ in FBS-free medium, cells were incubated for $24 \mathrm{~h}$ at $37^{\circ} \mathrm{C}$ in $10 \% \mathrm{FBS}$ medium.

Plasmid construction. The pre-miR-216a plasmid was constructed using the following synthetic oligonucleotides that were obtained from SangonBiotech, Shanghai, China: Sense, 5'-AATTCGATGGCTGTGAGTTGGCTTAATCTCAGCTG GCAACTGTGAGATGTTCATACAATCCCTCACAGTGGT CTCTGGGATTATGCTAAACAGAGCAATTTCCTAGCCC TCACGAA-3'; anti-sense, 5'-AGCTTTCGTGAGGGCTAGG AAATTGCTCTGTTTAGCATAATCCCAGAGACCACTGT GAGGGATTGTATGAACATCTCACAGTTGCCAGCT GAGACCAAGCCAACTCACAGCCATCG-3'. These oligonucleotides were then cloned into the pcDNA6.2-GW vector at the EcoRIand HindIII sites (Promega Corporation, Madison, WI, USA) using TargetScan, version 6.2 (www.targetscan. org/) to predict the target of miR-216a. Furthermore, the complimentary sites in the 3'-UTRs of the wild-type EIF4B (EIF4B-WT) and mutant EIF4B (EIF4B-MT) of miR-216a were synthesized (SangonBiotech) and cloned into the pmirGLO dual-luciferase reporter vector at the Sac1 and Xho 1 sites (Promega Corporation). The EIF4B expression plasmid was obtained from GeneCopoeia, Inc. (Rockville, MD, USA).

Reverse transcription-quantitative polymerase chain reaction $(R T-q P C R)$. The total RNA was isolated from the OSCC tissues and cells using TRIzol reagent (Invitrogen Life Technologies). All the reagents used for RT-qPCR were obtained from Tiangen Biotech Co., Ltd. (Beijing, China). For mRNA analyses, first-strand cDNA was synthesized using a FastQuant RT kit (Tiangen Biotech Co., Ltd.). The mature miRNA was reverse transcribed using specific primers for miR-216a. Subsequently, qPCR was performed using SuperReal PreMix Plus (SYBR Green) on an ABI7500 PCR machine (Applied Biosystems Life Technologies, Foster City, CA, USA). The relative expression levels of mRNA and miRNA were calculated based on the $2^{-\Delta \Delta \mathrm{Ct}}$ method. $\beta$-actin and U6 were used as controls for mRNA 1 and miRNA, respectively. The following primers that were synthesized by SangonBiotech were used in the qPCR analysis: RT 5'-GTCGTATCCAGTGCGTGTCGT GGAGTCGGCAATTGCACTGGATACGACTCACAGT-3' for miR-216a; miR216a, forward 5'-ATCCAGTGCGTGTCGTG-3' and reverse 5'-TGCTTAATCTCAGCTGGCA-3'; and EIF4B, forward 5'-AGCGTCAGCTGGATGAGCCAA-3' and reverse 5'-TGTCCTCGACCGTTCCCGTT-3'.
Cell proliferation assay. The transfected cells were seeded into 96-well plates at a density of 3,000 cells/well. At 24, 48 and $72 \mathrm{~h}$ following transfection, a 3-(4,5-dimethylthiazol-2-yl)-2,5-diphenyl tetrazolium bromide (MTT; Sigma-Aldrich, St. Louis, MO, USA) assay was used to determine the cell proliferation. The MTT solution $(5 \mathrm{mg} / \mathrm{ml} ; 200 \mu \mathrm{l})$ was added to the cells and incubated for $4 \mathrm{~h}$ at $37^{\circ} \mathrm{C}$. Following the removal of the culture medium, the remaining crystals were dissolved in $150 \mu 1$ dimethyl sulfoxide, and the absorbance at $492 \mathrm{~nm}$ was measured (FluoStar Optima; BD Biosciences, Franklin Lakes, NJ, USA).

Colony formation assay. The cells were cultured in 12-well plates at a density of 2,000 cells/well between $24 \mathrm{~h}$ and 15 days. The clones were washed with phosphate-buffered saline (PBS) and stained using $0.1 \%$ crystal violet (Beyotime Institute of Biotechnology, Haimen, China) for $20 \mathrm{~min}$. Images of the colonies were captured and the numbers of cells were counted under a microscope (CK2; Olympus, Tokyo, Japan).

Migration and invasion assays. The present study then performed cell migration and invasion assays using uncoated or coated Matrigel, respectively. Briefly, the cells $\left(5 \times 10^{4}\right)$ were added to the upper chamber of Transwell inserts in serum-free medium containing $0.1 \%$ FBS, and a medium supplemented with $10 \%$ FBS was added to the lower chamber. The cells were cultured for $24 \mathrm{~h}$ at $37^{\circ} \mathrm{C}$ in $5 \% \mathrm{CO}_{2}$. The Matrigel and non-invading cells were then gently removed, and the migrated and invaded cells in the lower membrane were fixed with $4 \%$ paraformaldehyde (Nanjing Chemical Material Corporation, Nanjing, China), stained using $0.1 \%$ crystal violet and counted under a light microscope (CK2; Olympus).

Dual luciferase reporter assays. The HEK 293 cells were seeded in 96-well plates (3,000 cells/well) and co-transfected with $0.1 \mathrm{mg}$ pmirGLO reporter plasmid (EIF4B-WT/EIF4B-MT) and $0.1 \mathrm{mg}$ pre-miR-216a plasmid or control miRNA. Cells were incubated at $37^{\circ} \mathrm{C}$ for $24 \mathrm{~h}$ and then lysed with a mixture of Dual-Glo Luciferase reagent and buffer (Promega Corporation). The luciferase activities were measured using the FluoStar Optima.

Western blot analysis. The total protein was extracted from the transfected cells using radioimmunoprecipitation assay lysis buffer (Beyotime Institute of Biotechnology, Haimen, China), according to the manufacturer's instructions. The total proteins $(50 \mu \mathrm{g})$ were separated using 10\% SDS-PAGE and transferred onto polyvinylidene difluoride membranes (EMD Millipore, Billerica, MA, USA), which were then blocked in $5 \%$ non-fat milk in Tris-buffered saline with $0.05 \%$ Tween-20 for $2 \mathrm{~h}$ at $37^{\circ} \mathrm{C}$, followed by incubation with rabbit monoclonal anti-EIF4B antibody $(1: 1,000 ; 17917-1-A P$; Protein Tech Group, Inc., Wuhan, China). $\beta$-actin was used as a loading control.

Statistical analysis. SPSS 13.0 software (SPSS, Inc., Chicago, IL, USA) was used for statistical analyses. All data are presented as the mean \pm standard error of the mean of at 

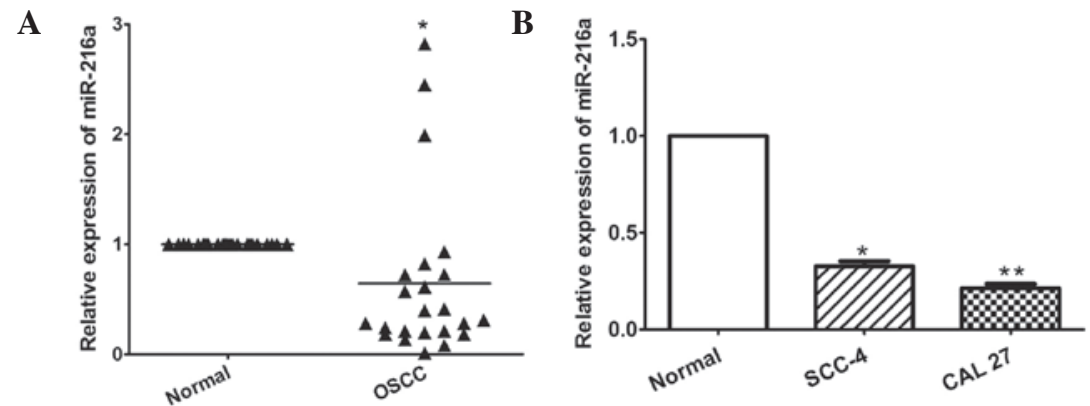

Figure 1. miR-216a is downregulated in OSCC tissues and cell lines. (A) Expression of miR-216a was significantly decreased in the OSCC tissues, compared with the adjacent normal tissues (determined using RT-qPCR). (B) Expression of miR-216a was significantly downregulated in the SCC-4 and CAL 27 cells, compared with the normal oral epithelial cells (determined using RT-qPCR). The data are presented as the mean \pm standard error of the mean (n=3). ${ }^{*} \mathrm{P}<0.05$ and ${ }^{* *} \mathrm{P}<0.01$, vs. control. OSCC, oral squamous cell carcinoma; RT-qPCR, reverse transcription-quantitative polymerase chain reaction.

A

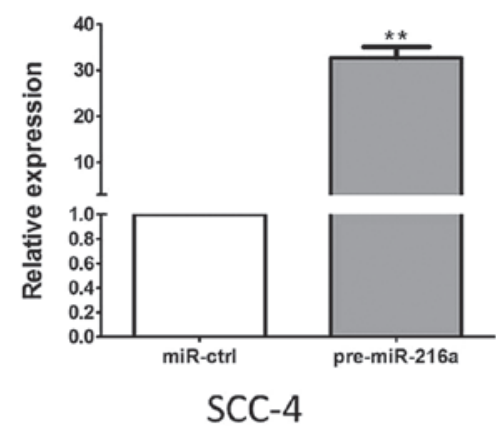

B

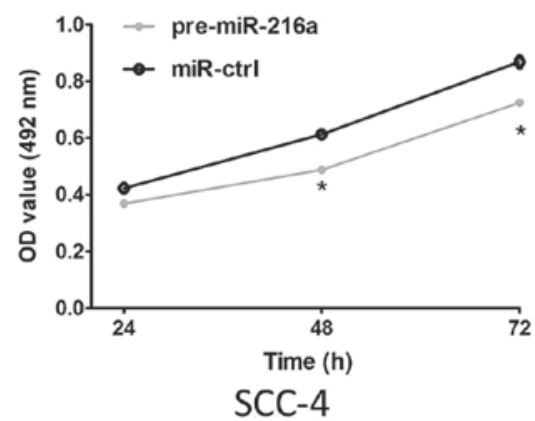

C

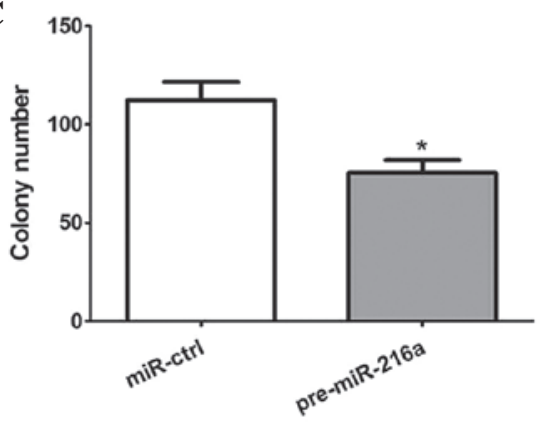

SCC-4
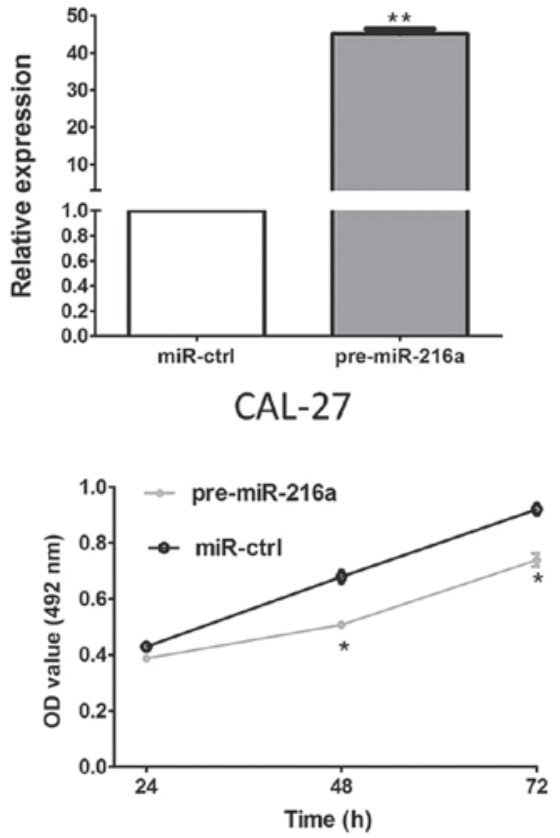

CAL-27

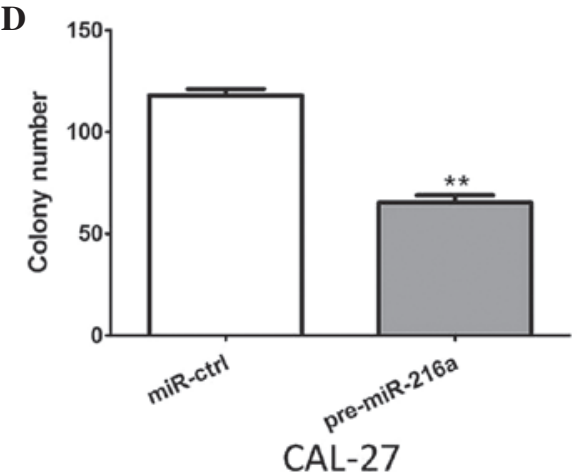

Figure 2. miR-216a suppresses the proliferation and colony formation of oral squamous cell carcinoma cells. (A) Expression levels of miR-216a in the SCC-4 and CAL 27 cells transfected with pre-miR-216a or miR-ctrl, determined using reverse transcription-quantitative polymerase chain reaction. (B) Cell proliferation was analyzed using a 3-(4,5-dimethylthiazol-2-yl)-2,5-diphenyl tetrazolium bromide assay in the SCC-4 and CAL 27 cells transfected with pre-miR-216a or miR-ctrl. Colony formation was analyzed in the (C) SCC-4 and (D) CAL 27 cells transfected with pre-miR-216a or miR-ctrl. Data are presented as the mean \pm standard error of the mean from three independent experiments. ${ }^{*} \mathrm{P}<0.05$ and ${ }^{* *} \mathrm{P}<0.01$, vs. miR-ctrl. miR-ctrl, empty-microRNA control; OD, optical density.

least three independent samples. The differences between the two groups were analyzed using Student's t-test. The linear correlation coefficient was calculated to estimate the correla- tion between miR-216a and EIF4B in the OSCC specimens. $\mathrm{P}<0.05$ was considered to indicate a statistically significant difference. 
A

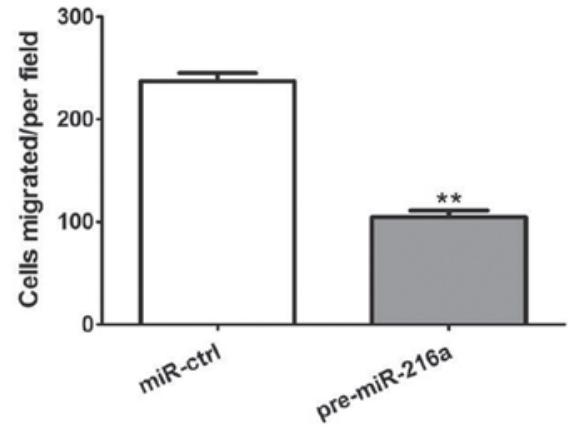

B

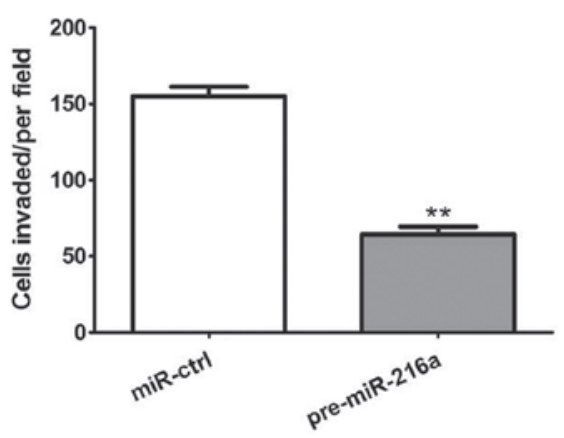

Figure 3. miR-216a suppresses metastasis of oral squamous cell carcinoma cells. (A) A migration assay was performed in the CAL 27 cells transfected with pre-miR-216a or miR-ctrl. (B) Invasion assay was performed in the CAL 27 cells transfected with pre-miR-216a or miR-ctrl. Data are presented as the mean \pm standard error of the mean from three independent experiments. ${ }^{* *} \mathrm{P}<0.01$, vs. miR-ctrl. miR-ctrl, empty-microRNA control.

\section{Results}

miR-216a is downregulated in OSCC tissues and cell lines. The present study first determined the expression of miR-216a using RT-qPCR in 23 OSCC tissues and in the SCC-4 and CAL 27 OSCC cell lines. As shown in Fig. 1A, miR-216a was significantly downregulated in the OSCC tissues compared with the adjacent normal tissues $(\mathrm{P}=0.034)$. Similarly, miR-216a was also decreased in the two OSCC cells, compared with the normal oral epithelial cells $(\mathrm{P}=0.002$ and $\mathrm{P}<0.001$, respectively; Fig. 1B).

miR-216a suppresses the growth of OSCC cells. The effect of miR-216a on the growth of OSCC cells was evaluated using MTT and colony formation assays. The results of the RT-qPCR analysis revealed that the expression levels of miR-26a in the SCC-4 and CAL 27 cells transfected with pre-miR-216a were significantly increased, compared with the control cells $(\mathrm{P}<0.001$; Fig. 2A). Furthermore, as shown in Fig. 2B, the overexpression of pre-miR-216a markedly inhibited the proliferation of the SCC-4 and CAL 27 cells $(\mathrm{P}<0.05)$. Consistent with these results, the overexpression of pre-miR-216a significantly suppressed the colony formation of the SCC-4 and CAL 27 cell lines $(\mathrm{P}=0.031$ and $\mathrm{P}<0.001$, respectively; Fig. 2C).

miR-216a suppresses the metastasis of OSCC cells. The present study subsequently investigated the effect of miR-216a on the metastasis of OSCC cells, including migration and invasion. As shown in Fig. 3A, the overexpression of pre-miR-216a significantly suppressed tumor cell migration in the CAL 27
A

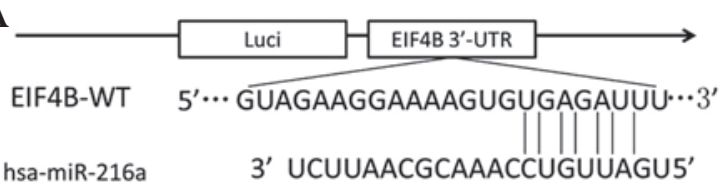

EIF4B-MT $\quad 5^{\prime} \cdots$ GUAGAAGGAAAAGUGCUGUUAGU $\cdots 3^{\prime}$

B

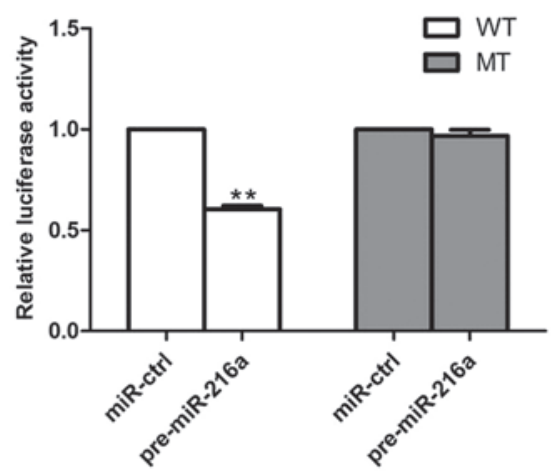

C

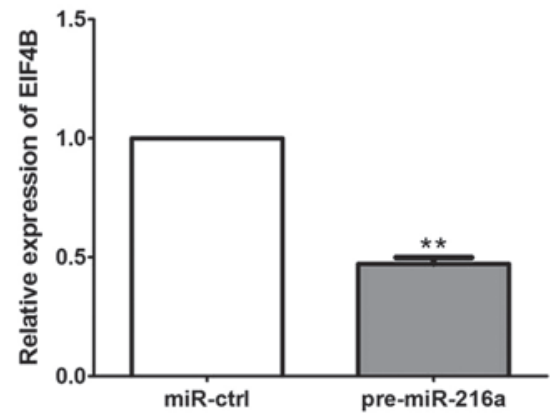

D

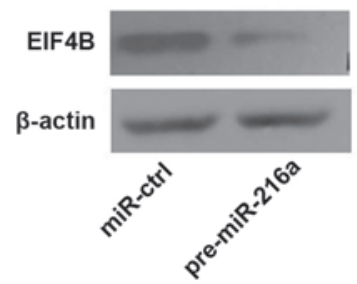

Figure 4. EIF4B is a target of miR-216a. (A) Predicted miR-216a binding site within the EIF4B 3'-UTR in the wild-type (WT) and mutated (MUT) type. (B) Relative luciferase activity was analyzed following co-transfection of the EIF4B plasmid with pre-miR-216a or miR-ctrl into HEK 293 cells (C) Reverse transcription-quantitative polymerase chain reaction and (D) western blot analyses were used to determine the effect of miR-216a on the mRNA and protein levels of EIF4B in the CAL 27 cells, respectively. Data are presented as the mean \pm standard error of the mean from three independent experiments. ${ }^{* *} \mathrm{P}<0.01$, vs. miR-ctrl. EIF4B, eukaryotic translation initiation factor 4B; miR-ctrl, empty-microRNA control; Luci, luciferase; UTR, untranslated region.

cells $(\mathrm{P}<0.001)$. In addition, the overexpression of pre-miR-216a markedly inhibited the invasive capacity of the CAL 27 cells $(\mathrm{P}<0.001$; Fig. 3B).

EIF4B is a direct target of miR-216a. The present study predicted that EIF4B may be a target of miR-216a using TargetScan 6.2. To confirm whether miR-216a directly targets EIF4B, as shown in Fig. 4A, EIF4B-WT and EIF4B-MT plasmids were constructed and cloned into the region downstream of the pmiRGLO dual-luciferase reporter vector. The subsequent 
A

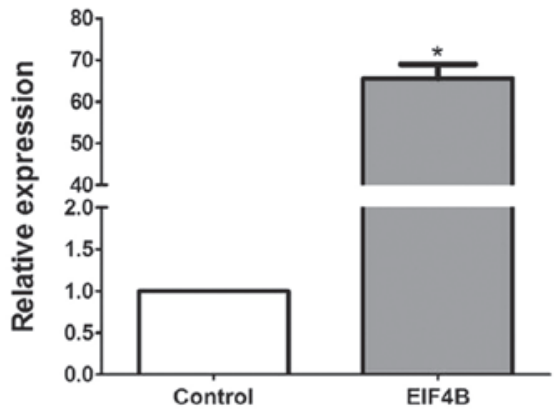

C

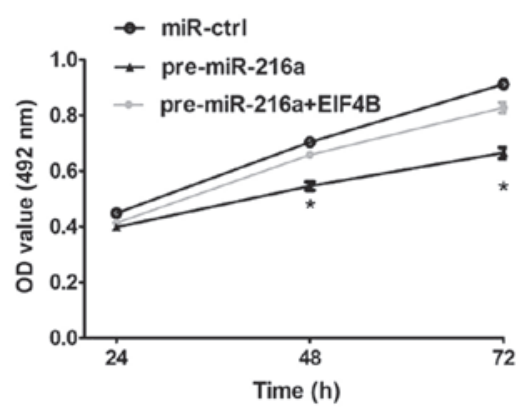

$\mathbf{E}$

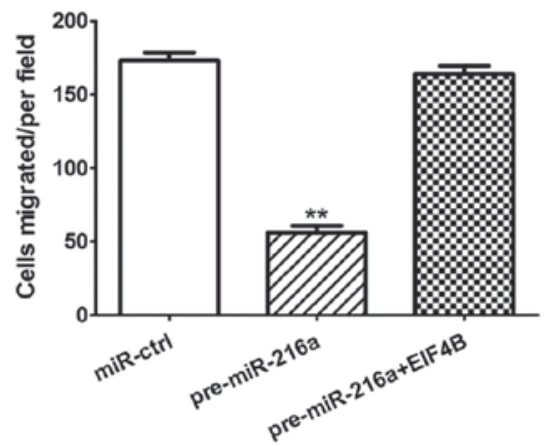

EIF4B

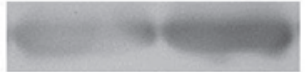

$\beta$-actin

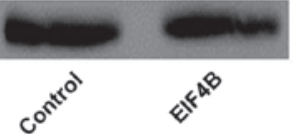

D

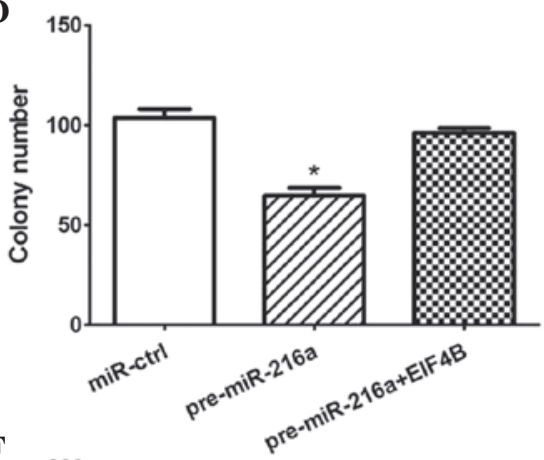

F

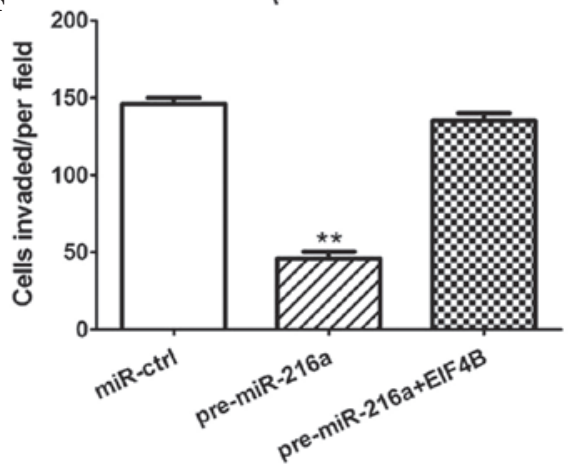

Figure 5. Overexpression of EIF4B attenuates the antitumor effect of miR-216a. (A) mRNA level of EIF4B in the CAL 27 cells transfected with EIF4B or the empty-pmirGLO vector (control). (B) Protein level of EIF4B in the CAL 27 cells transfected with EIF4B or the empty-pmirGLO vector(control), (C) A 3-(4,5-dimethylthiazol-2-yl)-2,5-diphenyl tetrazolium bromide assay was performed in the CAL 27 cells transfected with pre-miR-216a with or without the EIF4B overexpression plasmid. (D) Colony formation, (E) migration and (F) invasion assays were performed in the CAL 27 cells transfected with pre-miR-216a with or without the EIF4B overexpression plasmid. Data are presented as the mean \pm standard error of the mean from three independent experiments. ${ }^{*} \mathrm{P}<0.05$ and ${ }^{* *} \mathrm{P}<0.01$, vs. miR-ctrl. EIF4B, eukaryotic translation initiation factor 4B; miR-ctrl, empty-microRNA control; OD, optical density.
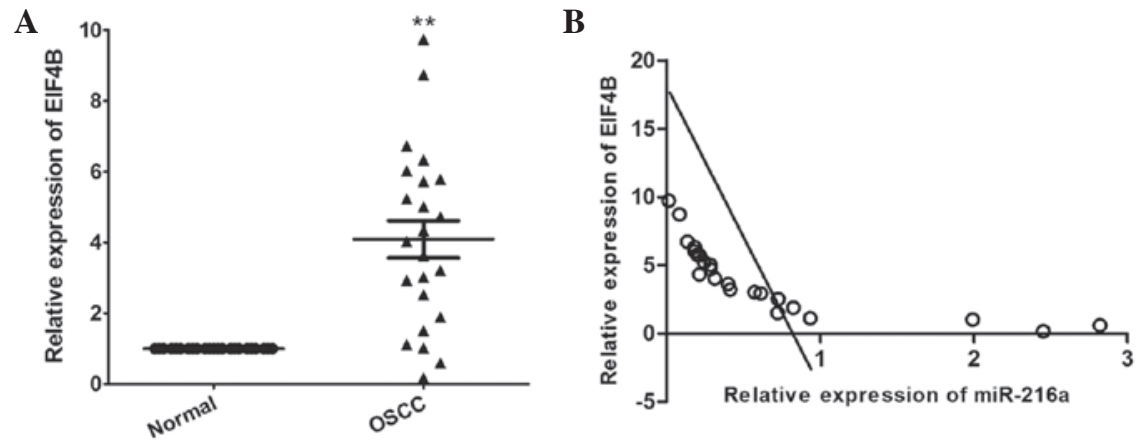

Figure 6. miR-216a is negatively correlated with EIF4B in OSCC tissues. (A) mRNA levels of EIF4B in the 23 pairs of OSCC and adjacent normal tissues were analyzed using reverse transcription-quantitative polymerase chain reaction. (B) Spearman's correlation analysis was used for the association between miR-216a and EIF4B. Data are presented as the mean \pm standard error of the mean from three independent experiments. "* P $<0.01$, vs. normal tissues. OSCC, oral squamous cell carcinoma; miR, microRNA; EIF4B, eukaryotic translation initiation factor 4B.

luciferase activity assay demonstrated that miR-216a significantly decreased luciferase activity of EIF4B-WT, but not EIF4B-MT in the HEK293 cells $(\mathrm{P}<0.001 ;$ Fig. 4B). In addition, the overexpression of pre-miR-216a in the CAL 27 cells significantly suppressed the mRNA and protein expression levels of EIF4B ( $\mathrm{P}<0.05$; Fig. 4C and D,). 
miR-216a inhibits the progression of OSCC cells by targeting $E I F 4 B$. In order to establish whether the overexpression of EIF4B attenuated the antitumor effects of miR-216a, an EIF4B overexpression plasmid was constructed, which significantly increased the expression of EIF4B at the mRNA and protein levels in the CAL 27 cells $(\mathrm{P}<0.05$; Fig. 5A and $\mathrm{B})$. Furthermore, the MTT, colony formation, migration and invasion assays demonstrated that the overexpression of EIF4B significantly attenuated the antitumor effects of miR-216a $(\mathrm{P}<0.05$; Fig. 5C-F).

Expression of miR-216a is inversely correlated with that of EIF4B in OSCC tissues. RT-qPCR was performed to determine the expression levels of EIF4B in OSCC and in matched adjacent normal tissues. As shown in Fig. 6A, the mRNA expression level of EIF4B was significantly increased in The OSCC tissues, compared with the adjacent normal tissues $(\mathrm{P}<0.001)$. In addition, the mRNA expression level of EIF4B was inversely correlated with that of miR-216a in the OSCC tissues ( $r=-0.761 ; \mathrm{P}<0.001$; Fig. 6B).

\section{Discussion}

The importance of miRNAs in tumor development have been identified, and miRNAs dysregulation can drive tumorigenesis as tumor suppressors or oncogenes in several types of tumor (11-14). The results of the present study provide important evidence, which supports miR-216a acting as a tumor suppressor in OSCC. In addition, the present study further identified EIF4B as a direct target of miR-216a, and the overexpression of EIF4B was observed to significantly attenuate the effects of miR-216a on suppressing the progression of OSCC.

Accumulating evidence has demonstrated that the dysfunction of miR-126a is involved in human malignancies and carcinogenesis, however, the detail mechanisms of miR-216a remain to be fully elucidated. In human hepatocellular carcinoma (HCC), miR-216a is significantly increased and contributes to early hepatocarcinogenesis through suppressing the gene expression of tumor suppressor in lung cancer-1 (15). Similarly, Xia et al also indicated that the expression of miR-216a was significantly upregulated in HCC tissue samples and cell lines, and was associated with early tumor recurrence and poor rates of disease-free survival. The overexpression of miR-216a-induced epithelial-mesenchymal transition activated the phosphatidylinositol 3-kinase/Akt and transforming growth factor- $\beta$ pathways and increased the stem-like cell population and migration and metastatic abilities of epithelial HCC cells by targeting phosphatase and tensin homolog and mothers against decapentaplegic homolog 7 (9). However, Wang et al demonstrated that the expression of miR-216a is downregulated in non-small cell lung cancer specimens, and the overexpression of miR-216a suppresses the growth and metastasis, and enhances cisplatin-induced cell growth inhibition and apoptosis in NSCLC cells (8). The results of the present study were consistent with those of Wang et al (8), and demonstrated the tumor suppressive role of miR-216a in the proliferation, colony formation, migration and invasion of OSCC cells. Therefore, it was suggested that miR-216a may have a unique pattern of expression in various types of cancer and function as an oncogene or suppressor gene.
EIF4B is comprised of eIF4A, eIF4B, eIF4E and eIF4G; and potentiates ribosome recruitment to the mRNA in the positioning of the ribosome over the start codon $(16,17)$. Deregulated translational control of the EIF4B complex members is considered to be important in oncogenic transformation (18-20). Horvilleur et al (18) demonstrated that the expression of EIF4B was increased in diffuse large B-cell lymphoma (BCL) patient samples. Reducing the expression of EIF4B alone was sufficient to decrease the synthesis of proteins associated with enhanced tumor cell survival, including excision repair cross-complementation group 5, death-associated protein 6 and BCL2; and the translational dysregulation of EIF4B may have resulted from aberrant signaling via the mammalian target of rapamycin pathway. In addition, Ren et al (19) demonstrated that the level of proviral integrations of moloney virus 2 (Pim-2) determined the phosphorylation level of EIF4B and the apoptotic rate of prostatic cells, suggesting that the overexpression of Pim-2 may inhibit the apoptosis of prostatic cells by phosphorylating EIF4B. In addition to the antiapoptotic effect of EIF4B on cancer cells, overexpression of EIF4B may also contribute to NSCLC cell growth and metastasis (8). In the present study, the 3'-UTR sequences of EIF4B were directly targeted by miR-216a, and EIF4B significantly attenuated the tumor suppressive effects of miR-216a on the OSCC cells. In addition, the expression of EIF4B was inversely correlated with miR-216a in OSCC tissues.

In conclusion, the present study demonstrated for the first time, to the best of our knowledge, that miR-216a, as a tumor suppressor, negatively suppressed the growth and metastasis of OSCC by targeting the 3'-UTR of EIF4B, suggesting that miR-216a may have important therapeutic potential for patients with OSCC.

\section{Acknowledgements}

The current study was supported by the Science Foundation of Shandong Province, China (grant no. ZR2014HL053).

\section{References}

1. Siegel R, Ma J, Zou Z and Jemal A: Cancer statistics, 2014. CA Cancer J Clin 64: 9-29, 2014.

2. DeSantis CE, Lin CC, Mariotto AB, et al: Cancer treatment and survivorship statistics, 2014. CA Cancer J Clin 64: 252-271, 2014.

3. Funk GF, Karnell LH, Robinson RA, Zhen WK, Trask DK and Hoffman HT: Presentation, treatment, and outcome of oral cavity cancer: a National Cancer Data Base report. Head Neck 24: 165-180, 2002.

4. Alsaleh G and Gottenberg JE: Characterization of microRNAs and their targets. Methods Mol Biol 1142: 55-63, 2014.

5. Di Leva $\mathrm{G}$ and Croce CM: miRNA profiling of cancer. Curr Opin Genet Dev 23: 3-11, 2013.

6. Bajan S and Hutvagner G: Regulation of miRNA Processing and miRNA Mediated Gene Repression in Cancer. Microrna 3: 10-17, 2014.

7. Wu BH, Xiong XP, Jia J and Zhang WF: MicroRNAs: new actors in the oral cancer scene. Oral Oncol 47: 314-319, 2011.

8. Wang RT, Xu M, Song ZG, Xu CX and Jin H: Decreased Expression of miR-216a Contributes to Non-small Cell Lung Cancer Progression. Clin Cancer Res 20: 4705-4716, 2014.

9. Xia H, Ooi LL and Hui KM: MicroRNA-216a/217-induced epithelial-mesenchymal transition targets PTEN and SMAD7 to promote drug resistance and recurrence of liver cancer. Hepatology 58: 629-641, 2013.

10. Hou B, Jian Z, Chen S, Ou Y, Li S and Ou J: Expression of miR-216a in pancreatic cancer and its clinical significance. Nan Fang Yi Ke Da Xue Xue Bao 32: 1628-1631, 2012 (In Chinese). 
11. Jia AY, Castillo-Martin M, Bonal DM, Sánchez-Carbayo M, Silva JM and Cordon-Cardo C: MicroRNA-126 inhibits invasion in bladder cancer via regulation of ADAM9. Br J Cancer 110: 2945-2954, 2014.

12. Zhang Z, Kim K, Li X, et al: MicroRNA-26b Represses Colon Cancer Cell Proliferation by Inhibiting Lymphoid Enhancer Factor 1 Expression. Mol Cancer Ther 13: 1942-1951, 2014.

13. Song Q, Xu Y, Yang C, et al: miR-483-5p promotes invasion and metastasis of lung adenocarcinoma by targeting RhoGDI1 and ALCAM. Cancer Res 74: 3031-3042, 2014.

14. Pan W, Wang H, Jianwei R and Ye Z: MicroRNA-27a promotes proliferation, migration and invasion by targeting MAP2K4 in human osteosarcoma cells. Cell Physiol. Biochem 33: 402-412, 2014.

15. Chen PJ, Yeh SH, Liu WH, et al: Androgen pathway stimulates microRNA-216a transcription to suppress the tumor suppressor in lung cancer-1 gene in early hepatocarcinogenesis. Hepatology 56 : 632-643, 2012.
16. Benne R and Hershey JW: The mechanism of action of protein synthesis initiation factors from rabbit reticulocytes. J Biol Chem 253: 3078-3087, 1978.

17. Schreier MH, Erni B and Staehelin T: Initiation of mammalian protein synthesis. I. Purification and characterization of seven initiation factors. J Mol Biol 116: 727-753, 1977.

18. Horvilleur E, Sbarrato T, Hill K, et al: A role for eukaryotic initiation factor 4B overexpression in the pathogenesis of diffuse large B-cell lymphoma. Leukemia 28: 1092-1102, 2014.

19. Ren K, Gou X, Xiao M, et al: The over-expression of Pim-2 promote the tumorigenesis of prostatic carcinoma through phosphorylating eIF4B. Prostate 73: 1462-1469, 2013.

20. Yang J, Wang J, Chen K, et al: eIF4B phosphorylation by pim kinases plays a critical role in cellular transformation by $\mathrm{Abl}$ oncogenes. Cancer Res 73: 4898-4908, 2013. 